\title{
Risk Analysis of Ports in Maritime Industry in Turkey using FMEA Based Intuitionistic Fuzzy TOPSIS Approach
}

\author{
Mine Şenel ${ }^{1}$, Bilgin Şenel ${ }^{1}$, Celal Alpay Havle ,** $^{*}$ \\ ${ }^{1}$ Munzur University, Department of Industrial Engineering, Tunceli, Turkey \\ ${ }^{2}$ Özyeğin University, Department of Pilot Training, İstanbul, Turkey
}

\begin{abstract}
Today, international trade is extremely important for countries' economies. It is possible to show the ship's transport as one of the most important execution channels of this trade. International commercial maritime transport subject to many rules and regulations carries many risks in processes such as loading, handling and unloading. Commercial ports are the places where these risks are seen intensely. From this point, many risk components in terms of the process in the port, one of the international commercial port in Turkey, based on a risk analysis is tried to be evaluated in this study. Especially, loading and unloading processes are focused with the directions of industrial experts. The main dimensions of the risks in the port are determined via expert opinions, and the sub-criteria of these dimensions are revealed. In this way, a generic model is proposed based on failure mode and effect analysis and the model is digitized using intuitionistic fuzzy TOPSIS. Interpretations are made in the direction of the obtained analysis results.
\end{abstract}

\section{Introduction}

Seaborne trade in world is vital for country economies in terms of balance of import and export. Nowadays, with $90 \%$ contribution to world trade, the importance of maritime industry has increased and to meet customer demands in the aspect of this, countries increase their number of vessels and facilities [1]. World marine trade fleet has reached 1.7 billion DWT [8] and maritime trade volume has reached 10.3 billion tons [1] and an estimated $\$ 500$ billion in revenue is generated annually from maritime trade. The vitality of well-structured and well-functioning international ports for industrial activities such as trade, globalized production processes should always be taken into consideration because ports have key role in global transport and trade chains [1].

Place of ports in global trade creates increased competitive forces and these influence the need for higher performance levels. These levels are not only related with cost

\footnotetext{
* Corresponding author: celal.alpay.havle@gmail.com
} 
reduction, optimization of operations, time efficiency and trade promotions but also security, safety, resource conservation, and environmental protection [1]. By considering the importance of international trade ports with the aspects mentioned above, port risks are focused in this study.

A port is a place that includes a terminal and the usual places where vessels are loaded and / or evacuated, loads and ships wait for their order, or wait or queue their order and it usually has an interface similar to other forms of transport and provides linking services in this way [5-6]. Within the context of increased transport concepts, port location is very important in the transport chain and to illustrate this concept in general terms, the diagram in Figure 1 is given [5-6]:

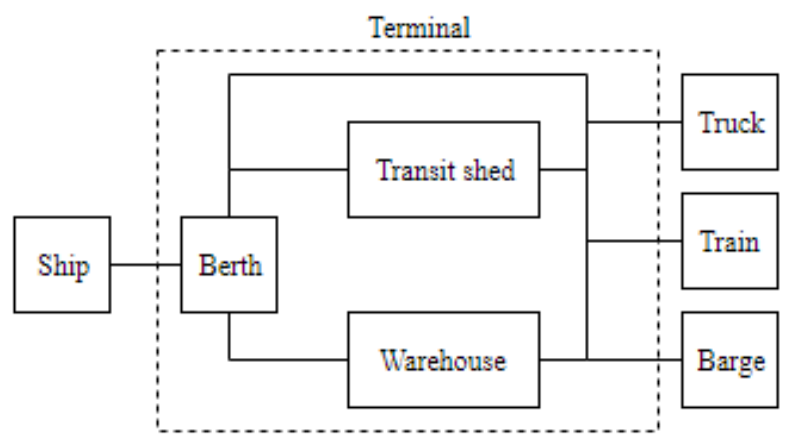

Figure 1. Port operations [5]

In this study, risks in the processes between ship and berth parts of port operations shown in Figure 1 are focused. Loading and unloading processed are examined with the industrial experts from Turkey's maritime industry. The study aims to determine the most important risk factors and failures in ports. To perform this failure mode and effect analysis is used to develop o model to construct a base for analyze. After construction of the model intuitionistic fuzzy TOPSIS approach is used.

The study contains following sections: Section 2 gives information about utilized methodology, section 3 gives numerical illustration of application of proposed methodology in Turkey maritime industry. By following this, section 4 highlights the obtained results before the conclusion.

\section{Methodology}

Failure mode and effect analysis (FMEA) is a technique to identify and potential failures, or errors in a system, process or design [14]. FMEA was developed as a formal design methodology by the aerospace industry for reliability and safety requirements in the 1960s [4-12].

The traditional FMEA determines the risk priorities of failure modes using the risk priority numbers (RPNs), which is defined as the probability of occurrence (O), severity (S) and detection (D) of a failure [17]. To calculate RPNs; O, S, and D of each failure should be multiplicated but, multiplication is not the right aggregation operator and FMEA factors must be aggregated in a nonlinear rather than linear manner [9]. The traditional FMEA has been well-accepted risk/criticality assesment method, however, it has been criticized for 
many drawbacks/shortcomings [4-7-10-11-12-13-16-17]. Significant criticisms include to the following:

- The relative importance among O,S,D is not taken into consideration in determining the priority of the failure modes. Three elements are assumed to have the same importance. However, this may not always be the case.

- Various sets of O,S and D may produce an identical value of RPN, however, the risk implication may be totally different. For example, two different failures with the $\mathrm{O}, \mathrm{S}$ and $\mathrm{D}$ values of 1,4,6 and 2,3,4,respectively, have the same RPN value of 24 . However, the risk implication is different.

- It is usually difficult or even impossible to give exact numerical evaluations of practically intangible quantities associated with the risk factors.

- The RPN scale is not continuous, with many holes because many numbers between 1 and 1000 cannot be obtained from the product of $\mathrm{O}, \mathrm{S}$, and $\mathrm{D}$.

- The three risk factors are difficult to be precisely evaluated. Much information in FMEA can be expressed linguistically in terms such as Likely, Important or Very high and so on.

To overcome obscurity during the evaluation process of risks and to address the above listed drawbacks, in this study Intuitionistic Fuzzy FMEA is conducted to assess risks in ports of Turkey. Intuitionistic fuzzy sets (IFS) are based on fuzzy set theory [19] but firstly introduced by Atanassov to eliminate obscurity [2]. Obscurity can be seen due to lack information in some cases [3-15]. In this study, FMEA model and TOPSIS method are augmented to IFSs [15] and an integrated intuitionistic fuzzy approach is applied to Turkey's maritime industry to analyze ports' risks.

Suppose that there are $n$ failure modes and they are evaluated by experts $E_{k}(1,2, \ldots, k)$ opinions based FMEA model. The model has risk factors and they are indicated as $C=\{O, S, D\}[15]$. These FMEA risk factors based on failure modes are evaluated with intuitionistic fuzzy sets. To perform this intuitionistic fuzzy TOPSIS (IF-TOPSIS) is applied. Steps of proposed approach are given as following [3-15]:

Step 1: Calculate the weight of experts: Suppose that there are $\mathrm{k}$ experts in the decision makers team. To calculate the weights intuitionistic fuzzy numbers are used and they are shown with Table 1 . Let $E_{k}=\left\{\mu_{k}, v_{k}, \pi_{k}\right\}$ be an intuitionistic fuzzy number (IFN) of $\mathrm{k}^{\text {th }}$ expert. To determine the weight of the expert formula (1) is used as follows:

$$
\lambda_{k}=\frac{\left(\mu_{k}+\pi_{k}\left(\frac{\mu_{k}}{\mu_{k}+v_{k}}\right)\right)}{\sum_{k=1}^{n}\left(\mu_{k}+\pi_{k}\left(\frac{\mu_{k}}{\mu_{k}+v_{k}}\right)\right)} \quad \text { where } \sum_{k=1}^{n} \lambda_{k}=1
$$


Table 1. Linguistic terms for importance of criteria and experts [15]

\begin{tabular}{lc}
\hline Linguistic terms & IFNs \\
\hline Very important & {$[0.9,0.1]$} \\
Important & {$[0.75,0.2]$} \\
Medium & {$[0.5,0.45]$} \\
Unimportant & {$[0.35,0.6]$} \\
Very unimportant & {$[0.1,0.9]$} \\
\hline
\end{tabular}

Step 2: Construct the aggregated intuitionistic fuzzy decision matrix based on experts' opinion: Let $R^{(k)}=\left(r_{i j}^{(k)}\right)_{m \times n}$ be the intuitionistic fuzz decision matrix of $\mathrm{k}^{\text {th }}$ experts and $\lambda=\left\{\lambda_{1}, \lambda_{2}, \lambda_{3}, \ldots, \lambda_{k}\right\}$ is the set of weights of each expert where $\sum_{k=1}^{l} \lambda_{k}=1$ and $\lambda_{k} \in[0,1]$. At the beginning of aggregation process, decision matrix of each experts should be constructed. To perform this, linguistic variables shown in Table 2 are used. To aggregate the decision matrices, IFWA operator [18] is used:

$$
\begin{aligned}
r_{i j} & =I F W A_{\lambda}\left(r_{i j}^{(1)}, r_{i j}^{(2)}, \ldots, r_{i j}^{(l)}\right)=\lambda_{1} r_{i j}^{(1)} \oplus \lambda_{2} r_{i j}^{(2)} \oplus \lambda_{3} r_{i j}^{(3)} \oplus \cdots \oplus \lambda_{l} r_{i j}^{(l)} \\
& =\left[1-\prod_{k=1}^{l}\left(1-\mu_{i j}{ }^{(k)}\right)^{\lambda_{k}}, \prod_{k=1}^{l}\left(v_{i j}{ }^{(k)}\right)^{\lambda_{k}}, \prod_{k=1}^{l}\left(1-\mu_{i j}{ }^{(k)}\right)^{\lambda_{k}}-\prod_{k=1}^{l}\left(v_{i j}{ }^{(k)}\right)^{\lambda_{k}}\right]
\end{aligned}
$$

where $r_{i j}=\left(\mu_{A_{i}}\left(x_{j}\right), v_{A_{i}}\left(x_{j}\right), \pi_{A_{i}}\left(x_{j}\right)\right)$ and $(i=1,2,3, \ldots, m, j=1,2,3, \ldots, n)$.

Table 2. Intuitionistic fuzzy linguistic variables [15]

\begin{tabular}{lclccc}
\hline \multicolumn{1}{c}{ Detectability } & \multicolumn{4}{c}{ Failure severity probability } & \multicolumn{2}{c}{ Failure occurrence probability } \\
\hline Linguistic terms & IFNs & Linguistic terms & IFNs & Linguistic terms & IFNs \\
\hline Absolutely impossible & {$[1,0]$} & Risky without warning & {$[1,0]$} & Very high & {$[0.9,0.1]$} \\
& & & & & \\
Highly unlikely & {$[0.9,0.1]$} & Risky with warning & {$[0.9,0.1]$} & High & {$[0.75,0.1]$} \\
Unlikely & {$[0.8,0.1]$} & Very high & {$[0.8,0.1]$} & Medium & {$[0.5,0.45]$} \\
Very low & {$[0.7,0.2]$} & High & {$[0.7,0.2]$} & Low & {$[0.35,0.6]$} \\
Low & {$[0.6,0.3]$} & Medium & {$[0.6,0.3]$} & Very low & {$[0.1,0.9]$} \\
Medium & {$[0.5,0.4]$} & Low & {$[0.5,0.4]$} & & \\
Relatively high & {$[0.4,0.5]$} & Very low & {$[0.4,0.5]$} & & \\
High & {$[0.25,0.6]$} & Insignificant & {$[0.25,0.6]$} & & \\
Very high & {$[0.1,0.75]$} & Very insignificant & {$[0.1,0.75]$} & & \\
Absolutely possible & {$[0.1,0.9]$} & None & {$[0.1,0.9]$} & & \\
\hline
\end{tabular}

Aggregated intuitionistic fuzzy decision matrix is obtained by following this aggregation process as follows: 


$$
\begin{gathered}
R=\left[\begin{array}{ccc}
\left(\mu_{F M_{1}}(O), v_{F M_{1}}(O), \pi_{F M_{1}}(O)\right) & \left(\mu_{F M_{1}}(S), v_{F M_{1}}(S), \pi_{F M_{1}}(S)\right) & \left(\mu_{F M_{1}}(D), v_{F M_{1}}(D), \pi_{F M_{1}}(D)\right) \\
\left(\mu_{F M_{2}}(O), v_{F M_{2}}(O), \pi_{F M_{2}}(O)\right) & \left(\mu_{F M_{2}}(S), v_{F M_{2}}(S), \pi_{F M_{2}}(S)\right) & \left(\mu_{F M_{2}}(D), v_{F M_{2}}(D), \pi_{F M_{2}}(D)\right) \\
\ddots & & \\
\left(\mu_{F M_{n}}(O), v_{F M_{n}}(O), \pi_{F M_{n}}(O)\right) & \left(\mu_{F M_{n}}(S), v_{F M_{n}}(S), \pi_{F M_{n}}(S)\right) & \left(\mu_{F M_{n}}(D), v_{F M_{n}}(D), \pi_{F M_{n}}(D)\right)
\end{array}\right] \\
=\left[\begin{array}{ccc}
r_{1 O} & r_{1 S} & r_{1 D} \\
r_{2 O} & r_{2 S} & r_{2 D} \\
\vdots & \ddots & \vdots \\
r_{n O} & r_{n S} & r_{n D}
\end{array}\right]
\end{gathered}
$$

Step 3: Calculate the weight of risk factors: Assume that $w_{j}^{k}=\left(\mu_{j}^{(k)}, v_{j}^{(k)}, \pi_{j}^{(k)}\right)$ is an intuitionistic fuzzy number assigned by $\mathrm{k}^{\text {th }}$ expert to $\mathrm{j}^{\text {th }}$ criterion, then the weights of risk factors are determined via IFWA operator [18] as follows:

$$
\begin{aligned}
w_{j} & =I F W A_{\lambda}\left(w_{j}^{(1)}, w_{j}^{(2)}, \ldots, w_{j}^{(l)}\right)=\lambda_{1} w_{j}^{(1)} \oplus \lambda_{2} w_{j}^{(2)} \oplus \lambda_{3} w_{j}^{(3)} \oplus \cdots \oplus \lambda_{l} w_{j}^{(l)} \\
& =\left[1-\prod_{k=1}^{l}\left(1-\mu_{j}{ }^{(k)}\right)^{\lambda_{k}}, \prod_{k=1}^{l}\left(v_{j}{ }^{(k)}\right)^{\lambda_{k}}, \prod_{k=1}^{l}\left(1-\mu_{j}{ }^{(k)}\right)^{\lambda_{k}}-\prod_{k=1}^{l}\left(v_{j}{ }^{(k)}\right)^{\lambda_{k}}\right]
\end{aligned}
$$

where $W=\left[w_{1}, w_{2}, w_{3}, \ldots, w_{j}\right], w_{j}=\left(\mu_{j}, v_{j}, \pi_{j}\right)$ and $(j=1,2, \ldots, n)$.

Step 4: Determine the weighted aggregated intuitionistic fuzzy decision matrix: Determination of the weight of each factor leads to aggregated decision matrix. To obtain this matrix following formulas (5) and (6) is used

$$
\begin{aligned}
& R \otimes W=\left\{\left\langle c, \mu_{F M_{i}}(c) \cdot \mu_{W}(c), v_{F M_{i}}(c)+v_{W}(c)-v_{F M_{i}}(c) \cdot v_{W}(c) \mid x \in X\right\rangle\right\} \\
& \pi_{F M_{i}}(c)=1-v_{F M_{i}}(c)-v_{W}(c)-\mu_{F M_{i}}(c) \cdot \mu_{W}(c)+v_{F M_{i}}(c) \cdot v_{W}(c)
\end{aligned}
$$

The weighted aggregated decision matrix is obtained as follows:

$$
R=\left[\begin{array}{ccc}
\left(\mu_{F M_{1} W}(O), v_{F M_{1} W}(O), \pi_{F M_{1} W}(O)\right) & \left(\mu_{F M_{1} W}(S), v_{F M_{1} W}(S), \pi_{F M_{1} W}(S)\right) & \left(\mu_{F M_{1} W}(D), v_{F M_{1} W}(D), \pi_{F M_{1} W}(D)\right) \\
\left(\mu_{F M_{2} W}(O), v_{F M_{2} W}(O), \pi_{F M_{2} W}(O)\right) & \left(\mu_{F M_{2} W}(S), v_{F M_{2} W}(S), \pi_{F M_{2} W}(S)\right) & \left(\mu_{F M_{2} W}(D), v_{F M_{2} W}(D), \pi_{F M_{2} W}(D)\right) \\
\ddots & \ddots & \\
\left(\mu_{F M_{n} W}(O), v_{F M_{n} W}(O), \pi_{F M_{n} W}(O)\right) & \left(\mu_{F M_{n} W}(S), v_{F M_{n} W}(S), \pi_{F M_{n} W}(S)\right) & \left(\mu_{F M_{n} W}(D), v_{F M_{n} W}(D), \pi_{F M_{n} W}(D)\right)
\end{array}\right]
$$

where $r_{i j}^{\prime}=\left(\mu_{i j}^{\prime}, v_{i j}^{\prime}, \pi_{i j}^{\prime}\right)=\left(\mu_{F M W}(c), v_{F M W}(c), \pi_{F M W}(c)\right)$.

Step 5: Determine the positive and negative ideal solutions based on IFNs: Let $J_{1}$ and $J_{2}$ are profit and cost criteria respectively. Then, positive and negative ideal solutions can be obtained by formulas (8) and (9) respectively:

where

$$
\begin{aligned}
& F M^{+}=\left(\mu_{F M^{+} W}\left(c_{j}\right), v_{F M^{+} W}\left(c_{j}\right)\right) \\
& F M^{-}=\left(\mu_{F M^{-} W}\left(c_{j}\right), v_{F M^{-} W}\left(c_{j}\right)\right)
\end{aligned}
$$

$$
\mu_{F M^{+} W}\left(c_{j}\right)=\left(\left\langle\max _{i} \mu_{F M_{i}, W}\left(c_{j}\right) \mid j \in J_{1}\right\rangle,\left\langle\min _{i} \mu_{F M_{i}, W}\left(c_{j}\right) \mid j \in J_{2}\right\rangle\right)
$$




$$
\begin{aligned}
& v_{F M^{+} W}\left(c_{j}\right)=\left(\left\langle\min _{i} v_{F M_{i}, W}\left(c_{j}\right) \mid j \in J_{1}\right\rangle,\left\langle\max _{i} v_{F M_{i}, W}\left(c_{j}\right) \mid j \in J_{1}\right\rangle\right) \\
& \mu_{F M^{-} W}\left(c_{j}\right)=\left(\left\langle\min _{i} \mu_{F M_{i}, W}\left(c_{j}\right) \mid j \in J_{1}\right\rangle,\left\langle\max _{i} \mu_{F M_{i}, W}\left(c_{j}\right) \mid j \in J_{2}\right\rangle\right) \\
& v_{F M^{-} W}\left(c_{j}\right)=\left(\left\langle\max _{i} v_{F M_{i}, W}\left(c_{j}\right) \mid j \in J_{1}\right\rangle,\left\langle\min _{i} v_{F M_{i}, W}\left(c_{j}\right) \mid j \in J_{1}\right\rangle\right)
\end{aligned}
$$

Step 6: Calculate the distances from positive and negative ideal solutions: To calculate separation measures normalized Euclidean distance is used as follows [3]:

$$
\begin{aligned}
& S^{+}=\sqrt{\frac{1}{2 n} \sum_{j=1}^{n}\left[\left(\mu_{F M_{i}, W}\left(c_{j}\right)-\mu_{F M^{+} W}\left(c_{j}\right)\right)^{2}+\left(v_{F M_{i}, W}\left(c_{j}\right)-v_{F M^{+} W}\left(c_{j}\right)\right)^{2}+\left(\pi_{F M_{i}, W}\left(c_{j}\right)-\pi_{F M^{+} W}\left(c_{j}\right)\right)^{2}\right]} \\
& S^{-}=\sqrt{\frac{1}{2 n} \sum_{j=1}^{n}\left[\left(\mu_{F M_{i}, W}\left(c_{j}\right)-\mu_{F M^{-} W}\left(c_{j}\right)\right)^{2}+\left(v_{F M_{i}, W}\left(c_{j}\right)-v_{F M^{-} W}\left(c_{j}\right)\right)^{2}+\left(\pi_{F M_{i}, W}\left(c_{j}\right)-\pi_{F M^{-} W}\left(c_{j}\right)\right)^{2}\right]}
\end{aligned}
$$

Step 7: Obtain the relative closeness coefficients based on separation measures:

$$
C_{i}^{+}=\frac{S_{i}^{-}}{S_{i}^{-}+S_{i}^{+}}
$$

\section{Numerical Illustration}

FMEA based IF-TOPSIS approach is performed in maritime industry in Turkey to analyze risks and their failure modes. Scope area is selected as ports loading and unloading areas with the directions of highly qualified industrial experts. Risk factors and their failure modes are determined with face to face interview and group decision. Constructed model and its sub-criteria are given in Table 3:

Table 3. FMEA model

\begin{tabular}{lll}
\hline & \multicolumn{2}{l}{ Dimensions and sub-criteria } \\
\hline Crane $\left(\mathrm{C}_{1}\right)$ & $\mathrm{FM}_{11}$ & Overloaded crane load limits \\
& $\mathrm{FM}_{12}$ & Shaking of crane control mechanisms \\
& $\mathrm{FM}_{13}$ & Hold damage with ropes \\
\hline Vessel Stress $\left(\mathrm{C}_{2}\right)$ & $\mathrm{FM}_{21}$ & Discharging sequence \\
& $\mathrm{FM}_{22}$ & Discrepancy of aft/forward drafts \\
& $\mathrm{FM}_{23}$ & Load positioning failures \\
\hline Vessel Performance $\left(\mathrm{C}_{3}\right)$ & $\mathrm{FM}_{31}$ & Hull (chest) condition \\
& $\mathrm{FM}_{32}$ & Misuse of fuel \\
& $\mathrm{FM}_{33}$ & Lack of maintenance \\
\hline & $\mathrm{FM}_{41}$ & Wing tank damage \\
& $\mathrm{FM}_{42}$ & Fuel tank damage \\
$\mathrm{FM}_{43}$ & Hatchway damage \\
Loading /Unloading $\left(\mathrm{C}_{4}\right)$ & $\mathrm{FM}_{44}$ & Equipment failures \\
\hline Personnel $\left(\mathrm{C}_{5}\right)$ & $\mathrm{FM}_{51}$ & Gang coordination failures \\
\hline
\end{tabular}




\begin{tabular}{lll} 
& $\mathrm{FM}_{52}$ & Knowledge level of crew \\
& $\mathrm{FM}_{53}$ & Neglect of technical personnel \\
\hline \multirow{3}{*}{ Weather $\left(\mathrm{C}_{6}\right)$} & $\mathrm{FM}_{61}$ & Separation \\
& $\mathrm{FM}_{62}$ & Manoeuver risks \\
& $\mathrm{FM}_{63}$ & Degradation of vision fields \\
& $\mathrm{FM}_{64}$ & Swell condition \\
\hline
\end{tabular}

Following the construction of FMEA model proposed methodology steps are performed. Weights of experts are given in Table 4, importance of risk factors are shown with Tables 5 and 6 as follows:

Table 4. Weights of experts

\begin{tabular}{|c|c|c|c|c|c|c|c|c|c|}
\hline Decision makers & \multicolumn{3}{|c|}{ Exp 1} & \multicolumn{3}{|c|}{ Exp2 } & \multicolumn{3}{|c|}{$\operatorname{Exp}_{3}$} \\
\hline Linguistic terms & & VI & & & I & & & M & \\
\hline IFNs & {$[0.9$} & 0.1 & 0] & {$[0.75$} & 0.2 & $0.05]$ & {$[0.5$} & 0.45 & $0.05]$ \\
\hline Weights & & 0.406 & & & 0.356 & & & 0.238 & \\
\hline
\end{tabular}

Table 5. Importance of $\mathrm{O}, \mathrm{S}$, D with linguistic variables

\begin{tabular}{cccc}
\hline FMEA Factors & Exp $_{1}$ & $\operatorname{Exp}_{2}$ & Exp $_{3}$ \\
\hline O & VI & I & I \\
S & M & I & M \\
D & I & VI & I \\
\hline
\end{tabular}

Table 6. Importance of O, S, D with IFNs

\begin{tabular}{ccccccccccc}
\hline FMEA Dimensions & \multicolumn{3}{c}{$\operatorname{Exp}_{1}$} & \multicolumn{3}{c}{$\operatorname{Exp}_{2}$} & \multicolumn{3}{c}{$\operatorname{Exp}_{3}$} \\
\hline $\mathrm{O}$ & {$[0.900$} & 0.100 & $0.000]$ & {$[0.750$} & 0.200 & $0.050]$ & {$[0.750$} & 0.200 & $0.050]$ \\
$\mathrm{S}$ & {$[0.500$} & 0.450 & $0.050]$ & {$[0.750$} & 0.200 & $0.050]$ & {$[0.500$} & 0.450 & $0.050]$ \\
$\mathrm{D}$ & {$[0.750$} & 0.200 & $0.050]$ & {$[0.900$} & 0.100 & $0.000]$ & {$[0.750$} & 0.200 & $0.050]$ \\
\hline
\end{tabular}

Experts' opinions are aggregated via IFWA operator and given with Table 7:

Table 7. Aggregated importance of O, S, D based on experts' opinion

\begin{tabular}{ccccccccc}
\hline & $\mathbf{O}$ & \multicolumn{3}{c}{$\mathbf{S}$} & \multicolumn{3}{c}{ D } \\
\hline$[0.828$ & 0.151 & $0.021]$ & {$[0.609$} & 0.337 & $0.054]$ & {$[0.819$} & 0.156 & $0.025]$ \\
\hline
\end{tabular}

From aggregated intuitionistic fuzzy decision matrix, positive and negative ideal solutions are obtained and shown in Table 8:

Table 8. Ideal solutions

\begin{tabular}{ccccccccccc}
\hline Ideal Solutions & \multicolumn{3}{c}{ O } & \multicolumn{3}{c}{ S } & \multicolumn{3}{c}{ D } \\
\hline $\mathrm{FM}^{+}$ & {$[0.516$} & 0.358 & $0.126]$ & {$[0.609$} & 0.337 & $0.054]$ & {$[0.558$} & 0.330 & $0.112]$ \\
$\mathrm{FM}^{-}$ & {$[0.175$} & 0.799 & $0.026]$ & {$[0.331$} & 0.573 & $0.096]$ & {$[0.082$} & 0.883 & $0.035]$ \\
\hline
\end{tabular}


Separation measures are calculated and used to obtained relative closeness coefficients and these are given in Table 9 below.

Table 9. Ideal solutions

\begin{tabular}{lccc}
\hline $\mathrm{FMs}$ & $\mathrm{Si}^{+}$ & $\mathrm{Si}^{-}$ & $\mathrm{C}_{\mathrm{i}}$ \\
\hline $\mathrm{FM}_{11}$ & 0.126 & 0.037 & 0.172 \\
$\mathrm{FM}_{12}$ & 0.128 & 0.045 & 0.208 \\
$\mathrm{FM}_{13}$ & 0.120 & 0.041 & 0.117 \\
$\mathrm{FM}_{21}$ & 0.119 & 0.047 & 0.049 \\
$\mathrm{FM}_{22}$ & 0.127 & 0.058 & 0.176 \\
$\mathrm{FM}_{23}$ & 0.082 & 0.080 & 0.363 \\
$\mathrm{FM}_{31}$ & 0.055 & 0.120 & 0.658 \\
$\mathrm{FM}_{32}$ & 0.094 & 0.109 & 0.620 \\
$\mathrm{FM}_{33}$ & 0.045 & 0.123 & 0.687 \\
$\mathrm{FM}_{41}$ & 0.103 & 0.082 & 0.649 \\
$\mathrm{FM}_{42}$ & 0.115 & 0.074 & 0.669 \\
$\mathrm{FM}_{43}$ & 0.124 & 0.095 & 0.180 \\
$\mathrm{FM}_{44}$ & 0.125 & 0.081 & 0.183 \\
$\mathrm{FM}_{51}$ & 0.094 & 0.074 & 0.214 \\
$\mathrm{FM}_{52}$ & 0.107 & 0.060 & 0.470 \\
$\mathrm{FM}_{53}$ & 0.049 & 0.136 & 0.793 \\
$\mathrm{FM}_{61}$ & 0.079 & 0.090 & 0.853 \\
$\mathrm{FM}_{62}$ & 0.115 & 0.082 & 0.369 \\
$\mathrm{FM}_{63}$ & 0.136 & 0.036 & 0.076 \\
$\mathrm{FM}_{64}$ & 0.121 & 0.090 & 0.064 \\
\hline & & &
\end{tabular}

\section{Results}

FMEA model in international trade ports is analyzed with IF-TOPSIS. Performing the steps of proposed methodology leads to determine the priority of failure modes. Determined relative closeness coefficients which are shown in Table 9, are used to prioritize these failures. This process is shown in Table 10:

Table 10. Ranked failure modes

\begin{tabular}{cllc}
\hline Ranked Order & & \multicolumn{1}{c}{ Failure Modes (Sub-criteria) } & $\mathrm{C}_{\mathrm{i}}$ \\
\hline 1 & $\mathrm{FM}_{53}$ & Neglect of technical personnel & 0.736 \\
2 & $\mathrm{FM}_{33}$ & Lack of maintenance & 0.732 \\
3 & $\mathrm{FM}_{31}$ & Hull (chest) condition & 0.686 \\
4 & $\mathrm{FM}_{32}$ & Misuse of fuel & 0.538 \\
5 & $\mathrm{FM}_{61}$ & Separation & 0.531 \\
6 & $\mathrm{FM}_{23}$ & Load positioning failures & 0.493
\end{tabular}




\begin{tabular}{ccll}
7 & $\mathrm{FM}_{41}$ & Wing tank damage & 0.443 \\
8 & $\mathrm{FM}_{51}$ & Gang coordination failures & 0.442 \\
9 & $\mathrm{FM}_{43}$ & Hatch coaming (Hatchway) damage & 0.434 \\
10 & $\mathrm{FM}_{64}$ & Swell condition & 0.426 \\
11 & $\mathrm{FM}_{62}$ & Manoeuver risks & 0.417 \\
12 & $\mathrm{FM}_{44}$ & Equipment failures & 0.394 \\
13 & $\mathrm{FM}_{42}$ & Fuel tank damage & 0.391 \\
14 & $\mathrm{FM}_{52}$ & Knowledge level of crew & 0.359 \\
15 & $\mathrm{FM}_{22}$ & Discrepancy of aft/forward drafts & 0.314 \\
16 & $\mathrm{FM}_{21}$ & Discharging sequence & 0.284 \\
17 & $\mathrm{FM}_{12}$ & Shaking of crane control mechanisms & 0.258 \\
18 & $\mathrm{FM}_{13}$ & Hold damage with ropes & 0.255 \\
19 & $\mathrm{FM}_{11}$ & Overloaded crane load limits & 0.226 \\
20 & $\mathrm{FM}_{63}$ & Degradation of vision fields & 0.211 \\
\hline
\end{tabular}

According to analysis results, first 5 most prone failure modes with higher priority in the failure of loading/unloading process at the ports are revealed as follows:

-Neglect of technical personnel,

-Lack of maintenance,

-Hull (chest) condition,

-Misuse of fuel,

-Separation.

Neglecting the known and applicable procedures negatively affects navigation safety. It affects the safety of the cargo carried and unintentional accidents cause injury and loss of personnel. Lack of maintenance of auxiliary machinery, generators, and other technical equipment can cause performance degradation and accidents. Maintenance deficiencies may cause the system to interfere with loading and unloading processes. Coverage of the bottom of the ship with moss and mussel, reduces the cruising speed and increases the fuel consumption. The reduction of cruising speed results in negative time in the planned cruising process. In addition, water-borne filters cause failure and failure of the required circulation. Like in land vehicles, marine fuels have different gravity values. It is also forbidden to use fuels with high sulfur content in some ports. Improper use of fuel increases consumption, causes pollution and reduces performance. The cargoes placed in the ship's warehouses are not properly separated, causing the cargo to slip under bad sea conditions. Loads are stacked on one side, risking the navigation and safety of the ship. It can even cause the ship to sink.

\section{Conclusion}

FMEA is a useful technique to analyze risks among different approaches. In this study, international trade ports in maritime industry is analyzed in terms of risks. To perform this, an FMEA model is constructed based in risk factors. Sub-criteria of these factors and their main dimensions are determined with experts' opinions. To eliminate obscurity and hesitancy during evaluation process intuitionistic fuzzy sets are used. IF-TOPSIS analytical tool is used to prioritize the failure modes and most important ones are determine. Study shows that FMEA based IF-TOPSIS methodology is useful for this application area. 
Constructed model is also proved that risks are evaluated in a hierarchical structure, so as a future work, analytic hierarchy process (AHP) can be used or different multi-criteria decision-making tools can be combined to reach more sensitive results.

\section{Acknowledgements}

Authors would like to give deep gratitude to industrial experts.

\section{References}

1. http://unctad.org/en/PublicationsLibrary/rmt2017_en.pdf

2. Atanassov, K. T. (1983). Intuitionistic fuzzy sets, VII ITKR's Session, Sofia deposed in Central Sci. Technical Library of Bulg. Acad. of Sci, 1697, 84.

3. Boran, F. E., Genç, S., Kurt, M., \& Akay, D. (2009). A multi-criteria intuitionistic fuzzy group decision making for supplier selection with TOPSIS method. Expert Systems with Applications, 36(8), 11363-11368.

4. Bowles, J. B., \& Pelaez, C. E. (1995). Fuzzy logic prioritization of failures in a system failure mode, effects and criticality analysis. Reliability Engineering and System Safety, 50, 203-213.

5. Branch, A.E. Elements of Port Operation and Management. Chapman and Hall. London (1986).

6. Branch, A.E. Elements of port operation and management. Springer Science \& Business Media (2012).

7. Gilchrist, W. (1993). Modelling Failure Modes and Effects Analysis. International Journal of Quality\& Reliability Management, 10(5), 16-23.

8. IMEAK Deniz Ticaret Odas1, Deniz Ticareti Dergisi, Şubat 2017, 1-64

9. Kutlu, A. C.,\& Ekmekçioğlu, M. (2012). Fuzzy failure modes and effects analysis by using fuzzy TOPSIS-based fuzzy AHP. Expert Sys. with Applications, 39(1), 61-67.

10. Liu, H.C., Liu, L. and Liu, N. ( 2013). Risk evaluation approaches in failure mode and effects analysis: A literature review, Expert Sys. with Applications, 40(2), 828-838.

11. Pillay, A., \& Wang, J. (2003). Modified failure mode and effects analysis using approximate reasoning. Reliability Engineering \& System Safety, 79, 69-85.

12. Sankar, N. R., \& Prabhu, B. S. (2001). Modified approach for prioritization of failures in a system failure mode and effects analysis. International Journal of Quality and Reliability Management, 18(3), 324-335.

13. Sharma,R.K., Kumar, D., \& Kumar, P. (2005). Systematic failure mode effect analysis (FMEA) using fuzzy linguistic modelling, International Journal of Quality \& Reliability Management, 22(9). 986-1004.

14. Stamatis, D. H. (1995). Failure mode and effect analysis: FMEA from theory to execution. Milwaukee, WI: ASQC Quality Press.

15. Tooranloo, H. S. \&Ayatollah, A.S. (2016). A model for failure mode and effects analysis based on intuitionistic fuzzy approach. Applied Soft Computing, 49, 238-247.

16. Ung, S.T., Williams, V. Bonsall, S. and Wang, J. (2009). The Risk Assessment and Management of Port Security Using Fuzzy Modeling, Marine Tech., 46(2). 61-73.

17. Wang, Y. M., Chin, K. S., Poon, G. K. K., \& Yang, J. B. (2009). Risk evaluation in failure mode and effects analysis using fuzzy weighted geometric mean. Expert Systems with Applications, 36, 1195-1207.

18. Xu, Z. (2007). Intuitionistic fuzzy aggregation operators. IEEE Transactions on fuzzy systems, 15(6), 1179-1187.

19. Zadeh, L. A. (1996). Fuzzy sets. In Fuzzy Sets, Fuzzy Logic, And Fuzzy Systems: Selected Papers by Lotfi A. Zadeh (pp. 394-432). 